\title{
Hybrid Parametric Optimality Constraints for Discrete Minmax Fractional Programming
}

\author{
Ram U Verma* \\ Department of Mathematics, University of North Texas, USA
}

Submission: April 29, 2017; Published: June 02, 2017

*Corresponding author: Ram U Verma, Department of Mathematics, University of North Texas, USA, Email: verma99@msn.com

\begin{abstract}
Several classes of generalized higher order parametric sufficient optimality constraints for a discrete minmax fractional programming problem are investigated toward establishing advanced results on higher order fractional programming. These results are established by applying advanced partitioning schemes and various types of generalized second-order $(F, \beta, \pi, \varphi, \rho, \theta, m)$-univexity assumptions. The obtained results are new and generalize most of the results on $(F, \beta, \varphi, \rho, \theta)$-univexity in the literature.
\end{abstract}

Keywords: Discrete minmax fractional programming; Second-order univex functions; Generalized sufficient optimality conditions

\section{Introduction}

In this paper, we intend to establish several sets of generalized parametric sufficient optimality conditions for the following discrete minmax fractional programming problem:

(P): Minimize $\max _{1 \leq i \leq p} \frac{f_{i}(x)}{g_{i}(x)}$

subject to $\mathrm{G}_{\mathrm{j}}(\mathrm{x}) \leq 0, j \in \underline{q}, \mathrm{H}_{\mathrm{k}}(\mathrm{x})=0, K \in \underline{r}, \mathrm{x} \in \mathrm{X}$,

where $\mathrm{X}$ is an open convex subset of $\mathbb{R}^{n}$ (n-dimensional Euclidean space), $\mathrm{f}_{\mathrm{i}^{\prime}} \mathrm{g}_{\mathrm{i}^{\prime}}, i \in \underline{p} \equiv\{1,2, \ldots, p\}, \mathrm{G}_{\mathrm{j}^{\prime}}, j \in \underline{q}$, and $\mathrm{H}_{\mathrm{k}^{\prime}}, K \in \underline{r}$ , are real-valued functions defined on X, and for each $j \in \underline{q}, \mathrm{~g}_{\mathrm{i}}$ $(\mathrm{x})>0$ for all $\mathrm{x}$ satisfying the constraints of $(\mathrm{P})$. Let $\mathrm{F}$ denote the feasible set (assumed to be nonempty) for (P) defined by

$$
\mathbb{F}=\{x \in X: G j(x) \leq 0, j \in \underline{q}, H k(x)=0, k \in \underline{r}\} .
$$

The present investigation is aimed at establishing various second-order necessary and sufficient optimality conditions for several types of optimization problems, using the generalized concepts of second-order invexity, pseudoinvexity, and quasiinvexity originally defined by Hanson [1], and a set of second-order necessary optimality conditions by introducing the new classes of generalized second-oder invex functions. We shall apply two partitioning schemes by Mond \& Weir [2] and Yang [3], in conjunction with the new classes of generalized secondorder invex functions to formulate and discuss numerous sets of generalized second-order sufficient optimality conditions for (P). To the best of our knowledge, all the second-order sufficient optimality results established in this paper are new in the area of discrete minmax fractional programming and encompass most of the investigations in the literature. The generalized optimality conditions established here can be utilized for constructing some generalized second-order parametric duality models for $(\mathrm{P})$ and proving numerous weak, strong, and strict converse duality theorems. For more details on the discrete minmax fractional programming and related literature, we refer the reader [1-9].

The rest of this paper is organized as follows. In the remainder of this section, we generalize a few basic definitions and recall some auxiliary results which will be needed in the sequel. In Section 2, we state and prove various second-order parametric sufficient optimality results for (P) using a variety of generalized $(F, \beta, \pi, \varphi, \rho, \theta, m)$-sounivexity assumptions. Finally, in Section 3 we summarize our main results and also point out some further research opportunities arising from certain modifications of the principal problem investigated in the present paper.

We next define some new classes of generalized secondorder univex functions, called (strictly) $(F, \beta, \varphi, \pi, \rho, \theta, m)$-sounivex, (strictly) $(\mathrm{F}, \beta, \varphi, \pi, \rho, \theta, \mathrm{m})$-pseudosounivex, and (pre-strictly) $(F, \beta, \varphi, \pi, \rho, \theta, m)$-quasisounivex functions. These are further extensions of the classes of second-order (strictly) $(\varphi, \eta, \rho, \theta, m)$ sonvex, (strictly) $(\varphi, \eta, \rho, \theta, m)-p s e u d o s o n v e x$, and (prestrictly) $(\varphi, \eta, \rho, \theta, m)$-quasisonvex functions which were introduced recently in [4]. The second-order univex functions are also referred to as "sounivex functions" in the litera-ture. For more on the generalized F-convex and other related functions, we 
refer the reader [8,9]. Now we present the new classes of $(\mathrm{F}, \beta, \pi, \varphi, \rho, \theta, \mathrm{m})$-sounivex functions at $\mathrm{X} *$. Let $f: X \rightarrow \mathbb{R}$ be a twice differentiable function.

\section{Definition}

The function $\mathrm{f}$ is said to be (strictly) $(\mathrm{F}, \beta, \pi, \varphi, \rho, \theta, \mathrm{m})$ sounivex at $\mathrm{x}^{*}$ if there exist functions $\beta: X \times X \rightarrow \mathbb{R}_{+} \equiv(0, \infty)$, $\varphi: \mathbb{R} \rightarrow \mathbb{R}, \quad \rho: X \times X \rightarrow R, \pi, \theta: X \times X \rightarrow \mathbb{R}^{n}$, a sublinear function $F\left(x, x^{*} ;\right): \mathbb{R}^{n} \rightarrow \mathbb{R}$, and a positive integer $\mathrm{m}$ such that for each $x \in X\left(x \neq x^{*}\right)$ and,$z \in \mathbb{R}^{n}$

$$
\begin{gathered}
\phi\left(f(x)-f\left(x^{*}\right)\right)(>) \geq F\left(x, x^{*} ; \beta\left(x, x^{*}\right) \nabla f\left(x^{*}\right)\right) \\
+\left\langle\pi\left(x, x^{*}\right), \nabla^{2} f\left(x^{*}\right) z\right\rangle \\
-\frac{1}{2}\left\langle z, \nabla^{2} f\left(x^{*}\right) z\right\rangle+\rho\left(x, x^{*}\right)\left\|\theta\left(x, x^{*}\right)\right\|^{m},
\end{gathered}
$$

where $\|\cdot\|$ is a norm on $\mathbb{R}^{n}$ and $\langle a, b\rangle$ is the inner product of the vectors $a$ and $b$. The function $\mathrm{f}$ is said to be (strictly) $(F, \beta, \pi, \varphi, \rho, \theta, m)$-sounivex on $X$ if it is (strictly) $(F, \beta, \pi, \varphi, \rho, \theta, m)$ sounivex at each $\mathrm{x}^{*} \in \mathrm{X}$.

\section{Definition}

The function $\mathrm{f}$ is said to be (strictly) $(\mathrm{F}, \beta, \varphi, \pi, \rho, \theta, \mathrm{m})$ pseudosounivex at $\mathrm{x}^{*}$ if there exist functions $\beta: X \times X \rightarrow \mathbb{R}_{+} \equiv(0, \infty)$, $\varphi: \mathbb{R} \rightarrow \mathbb{R}, \quad \rho: X \times X \rightarrow R, \pi, \quad \theta: X \times X \rightarrow \mathbb{R}^{n} \quad$ Hybrid optimality constraints a sublinear function $F\left(x, x^{*} ; \cdot\right): \mathbb{R}^{n} \rightarrow \mathbb{R}$, and a positive integer m such that for each $x \in X\left(x \neq x^{*}\right)$ and $z \in \mathbb{R}^{n}$,

$$
\begin{gathered}
F\left(x, x^{*} ; \beta\left(x, x^{*}\right) \nabla f\left(x^{*}\right)\right) \\
+\left\langle\pi\left(x, x^{*}\right), \nabla^{2} f\left(x^{*}\right) z\right\rangle \\
-\frac{1}{2}\left\langle z, \nabla^{2} f\left(x^{*}\right) z\right\rangle \\
\geq-\rho\left(x, x^{*}\right)\left\|\theta\left(x, x^{*}\right)\right\|^{m} \\
\Rightarrow \phi\left(f(x)-f\left(x^{*}\right)\right)(>) \geq 0 .
\end{gathered}
$$

The function $\mathrm{f}$ is said to be (strictly) $(\mathrm{F}, \beta, \pi, \varphi, \rho, \theta, \mathrm{m})$ pseudosounivex on $X$ if it is (strictly) $(F, \beta, \pi, \varphi, \rho, \theta, m)$ pseudosounivex at each $\mathrm{X}^{*} \in \mathrm{X}$.

\section{Definition}

The function $\mathrm{f}$ is said to be (prestrictly) $(\mathrm{F}, \beta, \pi, \varphi, \rho, \theta, \mathrm{m})$ quasisounivex at $\mathrm{X}^{*}$ if there exist functions $\beta: X \times X \rightarrow \mathbb{R}_{+} \equiv(0, \infty)$, $\varphi: \mathbb{R} \rightarrow \mathbb{R}, \quad \rho: X \times X \rightarrow R, \pi, \quad \theta: X \times X \rightarrow \mathbb{R}^{n}$, a sublinear function $F\left(x, x^{*} ;\right): \mathbb{R}^{n} \rightarrow \mathbb{R}$, and a positive integer $\mathrm{m}$ such that for each $\mathrm{x} \in \mathrm{X}$ and $z \in \mathbb{R}^{n}$,

$$
\begin{gathered}
\phi\left(f(x)-f\left(x^{*}\right)\right)(<) \leq 0 \Rightarrow \\
F\left(x, x^{*} ; \beta\left(x, x^{*}\right) \nabla f\left(x^{*}\right)\right) \\
+\left\langle\pi\left(x, x^{*}\right), \nabla^{2} f\left(x^{*}\right) z\right\rangle \\
-\frac{1}{2}\left\langle z, \nabla^{2} f\left(x^{*}\right) z\right\rangle \\
\leq-\rho\left(x, x^{*}\right)\left\|\theta\left(x, x^{*}\right)\right\|^{m} .
\end{gathered}
$$

The function $\mathrm{f}$ is said to be (prestrictly) $(F, \beta, \pi, \varphi, \rho, \theta, \mathrm{m})$ quasisounivex on $X$ if it is (prestrictly) $(F, \beta, \pi, \varphi, \rho, \theta, m)$ quasisounivex at each $\mathrm{X}^{*} \in \mathrm{X}$.

From the above definitions it is clear that if $\mathrm{fis}(\mathrm{F}, \beta, \pi, \varphi, \rho, \theta, \mathrm{m})$ sounivex at $x^{*}$, then it is both $(F, \beta, \pi, \varphi, \rho, \theta, m)$-pseudosounivex and $(F, \beta, \pi, \varphi, \rho, \theta, m)$-quasisounivex at $x^{*}$, if $f$ is $(F, \beta, \pi, \varphi, \rho, \theta, m)$ quasisounivex at $x^{*}$, then it is prestrictly $(F, \beta, \pi, \varphi, \rho, \theta, m)$ quasisounivex at $x^{*}$, and if $f$ is strictly $(F, \beta, \pi, \varphi, \rho, \theta, m)$ pseudosounivex at $x^{*}$, then it is $(F, \beta, \pi, \varphi, \rho, \theta, m)$-quasisounivex at $\mathrm{x}^{*}$.

In the proofs of the duality theorems, sometimes it may be more convenient to use certain alternative but equivalent forms of the above definitions. These are obtained by considering the contrapositive statements. For example, $(F, \beta, \pi, \varphi, \rho, \theta, m)$ quasisounivexity can be defined in the following equivalent way: The function $\mathrm{f}$ is said to be $(\mathrm{F}, \beta, \pi, \varphi, \rho, \theta, \mathrm{m})$-quasisounivex at $\mathrm{x}^{*}$ if there exist functions $\beta: X \times X \rightarrow \mathbb{R}_{+} \equiv(0, \infty), \varphi: \mathbb{R} \rightarrow \mathbb{R}, \rho: X \times X \rightarrow R, \pi$, $\theta: X \times X \rightarrow \mathbb{R}^{n}$, a sublinear function $F\left(x, x^{*} ; \cdot\right): \mathbb{R}^{n} \rightarrow \mathbb{R}$, and a positive integer $\mathrm{m}$ such that for each $\mathrm{x} \in \mathrm{X}$ and $z \in \mathbb{R}^{n}$,

$$
\begin{gathered}
F\left(x, x^{*} ; \beta\left(x, x^{*}\right) \nabla f\left(x^{*}\right)\right) \\
+\left\langle\pi\left(x, x^{*}\right), \nabla^{2} f\left(x^{*}\right) z\right\rangle \\
-\frac{1}{2}\left\langle z, \nabla^{2} f\left(x^{*}\right) z\right\rangle \\
\geq-\rho\left(x, x^{*}\right)\left\|\theta\left(x, x^{*}\right)\right\|^{m} \\
\Rightarrow \phi\left(f(x)-f\left(x^{*}\right)\right)(>) \geq 0 .
\end{gathered}
$$

We conclude this section by recalling a set of second-order parametric necessary optimality conditions for (P). The form and features of this result will provide clear guidelines for formulating various sets of second-order parametric sufficient optimality conditions for (P).

\section{Theorem}

[4] Let $\mathrm{x}^{*}$ be an optimal solution of $(\mathrm{P})$, let $\lambda^{*}=\phi\left(\mathrm{x}^{*}\right)$ be defined by

$$
\left.\lambda^{*}=\varphi\left(x^{*}\right) \equiv \max _{1 \leq i \leq p} f_{i}\left(x^{*}\right) / g_{i}\left(x^{*}\right)\right\},
$$

and assume that the functions $\mathrm{f}_{\mathrm{i}}, \mathrm{g}_{\mathrm{i}}, i \in \underline{p}, \mathrm{G}_{\mathrm{i}}, j \in \underline{q}$, and $\mathrm{H}_{\mathrm{k}^{\prime}}, k \in \underline{r}$, are twice continuously differentiable at $\mathrm{x}^{*}$, and that the second-order Guignard constraint qualification holds at $\mathrm{x}^{*}$. Then for each $\mathrm{z}^{*} \in \mathrm{C}\left(\mathrm{x}^{*}\right)$, there exist $u^{*} \in U \equiv\left\{u \in \mathbb{R}^{p}: u \geq 0, \sum_{i=1}^{p} u_{i}=1\right\}$, $v^{*} \in \mathbb{R}_{+}^{q} \equiv\left\{v \in \mathbb{R}^{q}: v \geq 0\right\}$ and $w^{*} \in \mathbb{R}^{r}$ such that

$$
\begin{aligned}
& \sum_{i=1}^{p} u_{i}^{*}\left[\nabla f_{i}\left(x^{*}\right)-\lambda^{*} \nabla g_{i}\left(x^{*}\right)\right]+\sum_{i=1}^{q} v_{j}^{*} \nabla G_{j}\left(x^{*}\right)+\sum_{k=1}^{r} w_{k}^{*} \nabla H_{k}\left(x^{*}\right)=0 \\
& \left\langle z^{*},\left\{\sum_{i=1}^{p} u_{i}^{*}\left[\nabla f_{i}\left(x^{*}\right)-\lambda^{*} \nabla g_{i}\left(x^{*}\right)\right]+\sum_{j=1}^{q} v_{j}^{*} \nabla G_{j}\left(x^{*}\right)+\sum_{k=1}^{r} w_{k}^{*} \nabla H_{k}\left(x^{*}\right)\right\} z^{*}\right\rangle \geq 0 \\
& u_{i}^{*}\left[f_{i}\left(x^{*}\right)-\lambda^{*} g_{i}\left(x^{*}\right)\right]=0, \quad, i \in \underline{p},
\end{aligned}
$$




$$
v_{j}^{*} G_{j}\left(x^{*}\right)=0, j \in \underline{q},
$$

where $\mathrm{C}\left(\mathrm{x}^{*}\right)$ is the set of all critical directions of $(\mathrm{P})$ at $\mathrm{x}^{*}$, that is,

$$
\begin{aligned}
& C\left(x^{*}\right)=\left\{z \in \mathbb{R}^{n}:\left\langle\nabla f_{i}\left(x^{*}\right)-\lambda \nabla g_{i}\left(x^{*}\right), z\right\rangle=0, \quad i \in A\left(x^{*}\right)\right. \\
& \left.\left\langle\nabla G_{j}\left(x^{*}\right), z\right\rangle \leq 0, j \in B\left(x^{*}\right),\left\langle\nabla H_{k}\left(x^{*}\right), z\right\rangle=0, k \in \underline{r}\right\}
\end{aligned}
$$

$A\left(x^{*}\right)=\left\{j \in \underline{p}: f_{j}\left(x^{*}\right) / g_{j}\left(x^{*}\right)=\max _{1 \leq i \leq p} f_{i}\left(x^{*}\right) / g_{i}\left(x^{*}\right)\right\}$, and $B\left(x^{*}\right)=\left\{j \in \underline{q}: G_{j}\left(x^{*}\right)=0\right\}$.

\section{Second-Order Sufficient Optimality}

In this section, we discuss several families of sufficient optimality results under various generalized $(F, \beta, \varphi, \pi, \rho, \theta, m)$ sounivexity hypotheses imposed on certain combinations of the problem functions. This is accomplished by employing a certain partitioning scheme which was originally proposed in [2] for the purpose of constructing generalized dual problems for nonlinear programming problems. For this we need some additional notation.

Let $\left\{\mathrm{J}_{0}, \mathrm{~J}_{1}, \ldots, \mathrm{J}_{\mathrm{M}}\right\}$ and $\left\{\mathrm{K}_{0}, \mathrm{~K}_{1}, \ldots, \mathrm{K}_{\mathrm{M}}\right\}$ be partitions of the index sets $\underline{q}$ and $\underline{r}$, respectively; thus, $J_{\mu} \subseteq \underline{q}$ for each $\mu \in \underline{M} \cup\{0\}$ , $J_{\mu} \cap J_{v}=\phi$ for each $\mu, v \in \underline{m} \cup\{0\}$ with $\mu \neq v$, and $U_{\mu=0}^{m} J_{\mu}=\underline{q}$ . Obviously, similar properties hold for $\left\{\mathrm{K}_{0}, \mathrm{~K}_{1}, \ldots, \mathrm{K}_{\mathrm{M}}\right\}$. Moreover, if $M_{1}$ and $M_{2}$ are the numbers of the partitioning sets of and, respectively, then $M=\max \left\{M_{1}, M_{2}\right\}$ and $J_{\mu}=\varnothing$ or $K_{\mu}=\emptyset$ for $\mu>$ $\min \left\{\mathrm{M}_{1}, \mathrm{M}_{2}\right\}$

In addition, we use the real-valued functions $\xi \rightarrow \Phi \mathrm{i}(\xi, \mathrm{v}, \mathrm{w}, \lambda)$, , $\xi \rightarrow \Phi(\xi, u, v, w, \lambda)$, and $\xi \rightarrow \Lambda t(\xi, v, w)$ defined, for fixed $u, v, w$, and $\lambda$ on $\mathrm{X}$ as follows:

$$
\begin{gathered}
\Phi_{i}(\xi, v, w, \lambda)=f_{i}(\xi)-\lambda_{i} g_{i}(\xi)+\sum_{j \in J_{0}} v_{j} G_{j}(\xi)+\sum_{k \in k_{0}} w_{k} H_{k}(\xi), i \in \underline{p} \\
\Phi_{i}(\xi, v, w, \lambda)=\sum_{i=1}^{p} u_{i}\left[f_{i}(\xi)-\lambda_{j} g_{i}(\xi)\right]+\sum_{j \in J_{0}} v_{j} G_{j}(\xi)+\sum_{k \in k_{0}} w_{k} H_{k}(\xi), \\
\Lambda_{t}(\xi, v, w)=\sum_{j \in J_{t}} v_{i} G_{j}(\xi)+\sum_{k \in K_{t}} w_{k} H_{k}(\xi), \quad t \in \underline{M} .
\end{gathered}
$$

In the proofs of our sufficiency theorems, we shall make frequent use of the following auxiliary result which provides an alternative expression for the objective function of $(\mathrm{P})$.

\section{Lemma}

For each $x \in X,[7]$

$$
\varphi(x)=\max _{1 \leq i \leq p} \frac{f_{i}(x)}{g_{i}(x)}=\max _{u \in U} \frac{\sum_{i=1}^{p} u_{i} f_{i}(x)}{\sum_{i=1}^{p} u_{i} g_{i}(x)} .
$$

Making use of the sets and functions defined above, we can now formulate our first collection of generalized second-order parametric sufficient optimality results for (P) as follows.

\section{Theorem}

Let $\mathrm{x}^{*} \in \mathrm{F}$, let $\lambda^{*}=\varphi\left(x^{*}\right)$, and assume that the functions $\mathrm{f}_{\mathrm{i}^{\prime}} \mathrm{g}_{\mathrm{i}^{\prime}}$ $i \in \underline{p}, \mathrm{G}_{\mathrm{j}^{\prime}} j \in \underline{q}$, and $\mathrm{H}_{\mathrm{k}^{\prime}}, k \in \underline{r}$ are twice differentiable at $\mathrm{x}^{*}$, and that for each there exist $u^{*} \in U, v^{*} \in \mathbb{R}_{+}$, and $w^{*} \in \mathbb{R}_{r}$ such that

$$
\begin{aligned}
& \sum_{i=1}^{p} u_{i}^{*}\left[\nabla f_{i}\left(x^{*}\right)-\lambda^{*} \nabla g_{i}\left(x^{*}\right)\right]+\sum_{j=1}^{q} v_{j}^{*} \nabla G_{j}\left(x^{*}\right)+\sum_{k=1}^{r} w_{k}^{*} \nabla H_{k}\left(x^{*}\right) z^{*}=0,(2.1) \\
& \left\langle\pi\left(x, x^{*}\right), \sum_{i=1}^{p} u_{i}^{*}\left[\nabla^{2} f_{i}\left(x^{*}\right)-\lambda^{*} \nabla^{2} g_{i}\left(x^{*}\right)\right] z^{*}+\sum_{j=1}^{q} v_{j}^{*} \nabla^{2} G_{j}\left(x^{*}\right) z^{*}+\sum_{k=1}^{r} w_{k}^{*} \nabla^{2} H_{k}\left(x^{*}\right) z^{*}\right\rangle \\
& \left\langle z^{*}, \sum_{i=1}^{p} u_{i}^{*}\left[\nabla^{2} f_{i}\left(x^{*}\right)-\lambda^{*} \nabla^{2} g_{i}\left(x^{*}\right) z^{*}+\sum_{j=1}^{q} v_{j}^{*} \nabla^{2} G_{j}\left(x^{*}\right) z^{*}+\sum_{k=1}^{r} w_{k}^{*} \nabla^{2} H_{k}\left(x^{*}\right) z^{*}\right\rangle\right. \\
& \geq 0 \forall x \in F(2.2) \\
& u_{i}^{*}\left[f_{i}\left(x^{*}\right)-\lambda^{*} g_{i}\left(x^{*}\right)\right]=0, \quad i \in \underline{p}(2.3) \\
& v_{j}^{*} G_{j}\left(x^{*}\right)=0 \quad i \in \underline{p}(2.4)
\end{aligned}
$$

Assume, furthermore, that any one of the following four sets of hypotheses is satisfied:

a.

1. $\xi \rightarrow \Phi\left(\xi, \mathrm{u}^{*}, \mathrm{v}^{*}, \mathrm{w}^{*}, \lambda^{*}\right)$ is prestrictly $(\mathrm{F}, \beta, \bar{\phi}, \pi, \bar{\rho}, \theta, \mathrm{m})-$ quasisounivex at $\mathrm{x}^{*}$ and $\bar{\phi}(\mathrm{a}) \geq 0 \Rightarrow \mathrm{a} \geq 0$;

2. For each , $\xi \rightarrow \Lambda \mathrm{t}\left(\xi, \mathrm{v}^{*}, \mathrm{w}^{*}\right)$ is strictly $\left(\mathrm{F}, \beta, \tilde{\phi}_{t}, \pi, \tilde{\rho}_{t}, \theta, \mathrm{m}\right)$ pseudosounivex at $x^{*}, \tilde{\phi}_{t}$ is increasing, and $\tilde{\phi}_{t}(0)=0$;

3. $\bar{\rho}\left(x, x^{*}\right)+\sum_{t=1}^{M} \tilde{\rho}_{t}\left(x, x^{*}\right) \geq 0$ for all $\mathrm{x} \in \mathrm{F}$;

b.

1. $\left(\xi \rightarrow \Phi\left(\xi, \mathrm{u}^{*}, \mathrm{v}^{*}, \mathrm{w}^{*}, \lambda^{*}\right)\right.$ is $(\mathrm{F}, \beta, \bar{\phi}, \bar{\rho}, \theta, \mathrm{m})$-pseudosounivex at $\mathrm{x}^{*}$ and $\bar{\phi}(\mathrm{a}) \geq 0 \Rightarrow \mathrm{a} \geq 0$;

2. For each , $\xi \rightarrow \Lambda_{\mathrm{t}}\left(\mathrm{z}, \mathrm{v}^{*}, \mathrm{w}^{*}\right)$ is $\left(\mathrm{F}, \beta, \tilde{\phi}_{t}, \pi, \tilde{\rho}_{t}, \theta, \mathrm{m}\right)$ quasisounivex $\tilde{\phi}_{t}$ at $\mathrm{x}^{*}$, is increasing, and $\tilde{\phi}_{t}(0)=0$;

3. $\bar{\rho}\left(x, x^{*}\right)+\sum_{t=1}^{M} \tilde{\rho}_{t}\left(x, x^{*}\right) \geq 0$ for all $\mathrm{x} \in \mathrm{F}$;

c.

1. $\xi \rightarrow \Phi\left(\xi, \mathrm{u}^{*}, \mathrm{v}^{*}, \mathrm{w}^{*}, \lambda^{*}\right)$ is prestrictly $(\mathrm{F}, \beta, \bar{\phi}, \pi, \bar{\rho}, \theta, \mathrm{m})-$ quasisounivex at $\mathrm{x}^{*} \bar{\phi}$ and $(\mathrm{a}) \geq 0 \Rightarrow \mathrm{a} \geq 0$;

2. For each , $\xi \rightarrow \Lambda_{\mathrm{t}}\left(\xi, \mathrm{v}^{*}, \mathrm{w}^{*}\right)$ is $\left(\mathrm{F}, \beta, \tilde{\phi}_{t}, \pi, \tilde{\rho}_{t}, \theta, \mathrm{m}\right)$ quasisounivex at $x^{*}, \tilde{\phi}$ is increasing, and $\tilde{\phi}(0)=0$;

3. $\bar{\rho}\left(x, x^{*}\right)+\sum_{t=1}^{M} \tilde{\rho}_{t}\left(x, x^{*}\right)>0$ for all $\mathrm{x} \in \mathrm{F}$;

d.

1. $\xi \rightarrow \Phi\left(\xi, \mathrm{u}^{*}, \mathrm{v}^{*}, \mathrm{w}^{*}, \lambda^{*}\right)$ is prestrictly $(\mathrm{F}, \beta, \bar{\phi}, \pi, \bar{\rho}, \theta, \mathrm{m})-$ quasisounivex at $\mathrm{x}^{*}$ and $(\mathrm{a}) \geq 0 \Rightarrow \mathrm{a} \geq 0$;

2. for each , $\xi \rightarrow \Lambda \mathrm{t}\left(\xi, \mathrm{V}^{*}, \mathrm{w}^{*}\right)$ is $\left(\mathrm{F}, \beta, \tilde{\phi}_{t}, \pi, \tilde{\rho}_{t}, \theta, \mathrm{m}\right)$ quasisounivex at $\mathrm{x}^{*}$, for each,$t \in \underline{M}_{2} \neq 0 \quad \xi \rightarrow \Lambda \mathrm{t}\left(\xi, \mathrm{v}^{*}, \mathrm{w}^{*}\right)$ is strictly $\left(\mathrm{F}, \beta, \tilde{\phi}_{t}, \pi, \tilde{\rho}_{t}, \theta, \mathrm{m}\right)-\quad \quad \quad$ pseudosounivex at $\mathrm{x}^{*}$, and for each $t \in \underline{M}, \tilde{\phi}_{t}$ is increasing and $\tilde{\phi}_{t}(0)=0$, where $\left\{\underline{M}_{1}, \underline{M}_{2}\right.$ \} is a partition of $\underline{M}$;

3. $\bar{\rho}\left(x, x^{*}\right)+\sum_{t=1}^{M} \tilde{\rho}_{t}\left(x, x^{*}\right) \geq 0$ for all $\mathrm{x} \in \mathrm{F}$.

Then $\mathrm{x}^{*}$ is an optimal solution of (P).

\section{Proof}

Let $\mathrm{x}$ be an arbitrary feasible solution of (P).

(a): In view of the sub linearity of $F\left(x, x^{*} ; \cdot\right)$, it is clear that 
(2.1) and (2.2) can be expressed as follows:

$$
\begin{aligned}
& F\left(x, x^{*} ; \beta\left(x, x^{*}\right)\left\{\sum_{i=1}^{p} u_{i}^{*}\left[\nabla f_{i}\left(x^{*}\right)-\lambda^{*} \nabla g_{i}\left(x^{*}\right)\right]+\sum_{i \in J_{0}} v_{j}^{*} \nabla G_{j}\left(x^{*}\right)+\sum_{k \in K_{0}} \omega_{k}^{*} \nabla H_{k}\left(x^{*}\right)\right\}\right) \\
& +F\left(x, x^{*} ; \beta\left(x, x^{*}\right) \sum_{t=1}^{M}\left[\sum_{i \in J_{t}} v_{j}^{*} \nabla G_{j}\left(x^{*}\right)+\sum_{k \in k_{t}} \omega_{k}^{*} \nabla H_{k}\left(x^{*}\right)\right]\right) \geq 0(2.5) \\
& \left\langle\pi\left(x, x^{*}\right), \sum_{i=1}^{p} u_{i}^{*}\left[\nabla^{2} f_{i}\left(x^{*}\right)-\lambda^{*} \nabla^{2} g_{i}\left(x^{*}\right)\right] z^{*}+\sum_{j=1}^{q} v_{j}^{*} \nabla^{2} G_{j}\left(x^{*}\right) z^{*}+\sum_{k=1}^{r} w_{k}^{*} \nabla^{2} H_{k}\left(x^{*}\right) z^{*}\right\rangle \\
& +\left\langle\pi\left(x, x^{*}\right), \sum_{t=1}^{M}\left[\sum_{j \in J_{t}} v_{j}^{*} \nabla^{2} G_{j}\left(x^{*}\right)+\sum_{k \in K_{t}} w_{k}^{*} \nabla^{2} H_{k}\left(x^{*}\right)\right] z^{*}\right\rangle \\
& -\frac{1}{2}\left[\left\langle z^{*}, \sum_{i=1}^{p} u_{i}^{*}\left[\nabla^{2} f_{i}\left(x^{*}\right)-\lambda^{*} \nabla^{2} g_{i}\left(x^{*}\right) z^{*}+\sum_{j=1}^{q} v_{j}^{*} \nabla^{2} G_{j}\left(x^{*}\right) z^{*}+\sum_{k=1}^{r} w_{k}^{*} \nabla^{2} H_{k}\left(x^{*}\right) z^{*}\right\rangle\right]\right. \\
& +\left\langle z^{*}, \sum_{t=1}^{M}\left[\sum_{j \in J_{t}} v_{j}^{*} \nabla^{2} G_{j}\left(x^{*}\right)+\sum_{k \in K_{t}} w_{k}^{*} \nabla^{2} H_{k}\left(x^{*}\right)\right] z^{*}\right\rangle \geq 0
\end{aligned}
$$

Since for each $t \in \underline{M}$, of $x$ )

$\Lambda_{t}\left(x, v^{*}, w^{*}\right)=\sum_{j \in J_{t}} v_{j}^{*} G_{j}(x)+\sum_{k \in K_{t}} w_{k}^{*} H_{k}(x) \leq 0 \quad$ (by the feasibility

$$
\sum_{j \in J_{t}} v_{j}^{*} G_{j}(x)+\sum_{k \in K_{t}} w_{k}^{*} H_{k}\left(x^{*}\right)
$$

(by (2.4) and the feasibility of $x^{*}$ )

$$
=\Lambda_{t}\left(x^{*}, v^{*}, w^{*}\right) \text {, }
$$
that

and hence $\tilde{\phi}_{t}\left(\Lambda_{t}\left(x^{*}, v^{*}, w^{*}\right)-\Lambda_{t}\left(x^{*}, v^{*}, w^{*}\right)\right) \leq 0$, it follows from (ii)

$$
\begin{aligned}
& F\left(x, x^{*} ; \beta\left(x, x^{*}\right)\left[\sum_{j \in J_{t}} v_{j}^{*} G_{j}(x)+\sum_{k \in K_{t}} w_{k}^{*} H_{k}(x)\right]\right) \\
+ & \left\langle\pi\left(x, x^{*}\right),\left[\sum_{j \in J_{t}} v_{j}^{*} G_{j}(x)+\sum_{k \in K_{t}} w_{k}^{*} H_{k}(x)\right] z^{*}\right\rangle \\
& -\frac{1}{2}\left\langle z^{*},\left[\sum_{j \in J_{t}} v_{j}^{*} G_{j}(x)+\sum_{k \in K_{t}} w_{k}^{*} H_{k}(x)\right] z^{*}\right\rangle \\
< & -\tilde{\rho}_{t}\left(x, x^{*}\right)\left\|\theta\left(x, x^{*}\right)\right\|^{m}
\end{aligned}
$$

Summing over $t \in \underline{M}$ and using the sublinearity of $F\left(x, x^{*} ; \cdot\right)$ , we obtain

$$
\begin{aligned}
& F\left(x, x^{*} ; \beta\left(x, x^{*}\right) \sum_{t=1}^{M}\left[\sum_{i \in J_{t}} v_{j}^{*} \nabla G_{j}\left(x^{*}\right)+\sum_{k \in k_{t}} \omega_{k}^{*} \nabla H_{k}\left(x^{*}\right)\right]\right) \\
& +\left\langle\pi\left(x, x^{*}\right), \sum_{t=1}^{M}\left[\sum_{i \in J_{t}} v_{j}^{*} \nabla^{2} G_{j}\left(x^{*}\right)+\sum_{k \in k_{t}} \omega_{k}^{*} \nabla^{2} H_{k}\left(x^{*}\right)\right] z^{*}\right\rangle \\
& -\frac{1}{2}\left\langle z^{*}, \sum_{t=1}^{M}\left[\sum_{i \in J_{t}} v_{j}^{*} \nabla^{2} G_{j}\left(x^{*}\right)+\sum_{k \in k_{t}} \omega_{k}^{*} \nabla^{2} H_{k}\left(x^{*}\right)\right] z^{*}\right\rangle \\
& <-\sum_{t=1}^{M} \tilde{\rho}_{t}\left(x, x^{*}\right)\left\|\theta\left(x, x^{*}\right)\right\|^{m} .
\end{aligned}
$$

Combining (2.5)-(2.7), and using (iii) we get $F\left(x, x^{*} ; \beta\left(x, x^{*}\right)\left\{\sum_{i=1}^{p} u_{i}^{*}\left[\nabla f_{i}\left(x^{*}\right)-\lambda^{*} \nabla g_{i}\left(x^{*}\right)\right]+\sum_{i \in J_{0}} v_{j}^{*} \nabla G_{j}\left(x^{*}\right)+\sum_{k \in K_{0}} \omega_{k}^{*} \nabla H_{k}\left(x^{*}\right)\right\}\right)$

$$
\begin{aligned}
& +\left\langle\pi\left(x, x^{*}\right), \sum_{i=1}^{p} u_{i}^{*}\left[\nabla^{2} f_{i}\left(x^{*}\right)-\lambda^{*} \nabla^{2} g_{i}\left(x^{*}\right)\right]+\sum_{i \in J_{t}} v_{j}^{*} \nabla^{2} G_{j}\left(x^{*}\right)+\sum_{k \in k_{t}} \omega_{k}^{*} \nabla^{2} H_{k}\left(x^{*}\right) z^{*}\right\rangle \\
& -\frac{1}{2}\left\langle z^{*},\left\{\sum_{i=1}^{p} u_{i}^{*}\left[\nabla^{2} f_{i}\left(x^{*}\right)-\lambda^{*} \nabla^{2} g_{i}\left(x^{*}\right)\right]+\sum_{i \in J_{t}} v_{j}^{*} \nabla^{2} G_{j}\left(x^{*}\right)+\sum_{k \in k_{t}} \omega_{k}^{*} \nabla^{2} H_{k}\left(x^{*}\right)\right\} z^{*}\right\rangle \\
& >-\sum_{t=1}^{M} \tilde{\rho}_{t}\left(x, x^{*}\right)\left\|\theta\left(x, x^{*}\right)\right\|^{m} \geq-\bar{\rho}\left(x, x^{*}\right)\left\|\theta\left(x, x^{*}\right)\right\|^{m},(2.8)
\end{aligned}
$$

which by virtue of (i) implies that

$\bar{\phi}\left(\Phi\left(x, u^{*}, v^{*}, w^{*}, \lambda^{*}\right)-\Phi\left(x, u^{*}, v^{*}, w^{*}, \lambda^{*}\right)\right) \geq 0$.

But $\bar{\phi}(\mathrm{a}) \geq 0 \Rightarrow \mathrm{a} \geq 0$, and hence we get

$\Phi\left(x, u^{*}, v^{*}, w^{*}, \lambda^{*}\right) \geq \Phi\left(x, u^{*}, v^{*}, w^{*}, \lambda^{*}\right)=0$

where the equality follows from (2.3), (2.4), and the feasibility of $x^{*}$. Since $x \in F$, the above inequality reduces to

$$
\sum_{i=1}^{p} u_{i}^{*}\left[f_{i}(x)-\lambda^{*} g_{i}(x)\right] \geq 0(2.9)
$$

Now using (2.9) and Lemma 2.1, we see that

$$
\varphi\left(x^{*}\right)=\lambda^{*} \leq \frac{\sum_{i=1}^{p} u_{i}^{*} f_{i}(x)}{\sum_{i=1}^{p} u_{i}^{*} g_{i}(x)} \leq \max _{u \in U} \frac{\sum_{i=1}^{p} u_{i} f_{i}(x)}{\sum_{i=1}^{p} u_{i} g_{i}(x)}=\varphi(x) .
$$

Since $x \in F$ was arbitrary, we conclude from this inequality that $\mathrm{X} *$ is an optimal solution of $(\mathrm{P})$.

(b) : Proceeding as in the proof of part (a), we see that (ii) leads to the following inequality:

$$
\begin{gathered}
F\left(x, x^{*} ; \beta\left(x, x^{*}\right) \sum_{t=1}^{M}\left[\sum_{j \in J_{t}} v_{j}^{*} \nabla^{2} G_{j}\left(x^{*}\right)+\sum_{k \in K_{t}} w_{k}^{*} \nabla^{2} H_{k}\left(x^{*}\right)\right]\right) \\
+\left\langle\pi\left(x, x^{*}\right), \sum_{t=1}^{M}\left[\sum_{j \in J_{t}} v_{j}^{*} \nabla^{2} G_{j}\left(x^{*}\right)+\sum_{k \in K_{t}} w_{k}^{*} \nabla^{2} H_{k}\left(x^{*}\right)\right] z^{*}\right\rangle \\
-\frac{1}{2}\left\langle z^{*}, \sum_{t=1}^{M}\left[\sum_{j \in J_{t}} v_{j}^{*} \nabla^{2} G_{j}\left(x^{*}\right)+\sum_{k \in K_{t}} w_{k}^{*} \nabla^{2} H_{k}\left(x^{*}\right)\right] z^{*}\right\rangle \leq-\sum_{t=1}^{M} \tilde{\rho}_{t}\left(x, x^{*}\right)\left\|\theta\left(x, x^{*}\right)\right\|^{m} \\
\text { Combining this inequality with (2.6) and (2.7), and using }
\end{gathered}
$$
(iii) we get

$$
\begin{aligned}
& F\left(x, x^{*} ; \beta\left(x, x^{*}\right)\left\{\sum_{i=1}^{p} u_{i}^{*}\left[\nabla f_{i}\left(x^{*}\right)-\lambda^{*} \nabla g_{i}\left(x^{*}\right)\right]+\sum_{i \in J_{0}} v_{j}^{*} \nabla G_{j}\left(x^{*}\right)+\sum_{k \in K_{0}} \omega_{k}^{*} \nabla H_{k}\left(x^{*}\right)\right\}\right) \\
& +\left\langle\pi\left(x, x^{*}\right), \sum_{i=1}^{p} u_{i}^{*}\left[\nabla^{2} f_{i}\left(x^{*}\right)-\lambda^{*} \nabla^{2} g_{i}\left(x^{*}\right)\right]+\sum_{i \in J_{t}} v_{j}^{*} \nabla^{2} G_{j}\left(x^{*}\right)+\sum_{k \in k_{t}} \omega_{k}^{*} \nabla^{2} H_{k}\left(x^{*}\right) z^{*}\right\rangle \\
& -\frac{1}{2}\left\langle z^{*},\left\{\sum_{i=1}^{p} u_{i}^{*}\left[\nabla^{2} f_{i}\left(x^{*}\right)-\lambda^{*} \nabla^{2} g_{i}\left(x^{*}\right)\right]+\sum_{i \in J_{t}} v_{j}^{*} \nabla^{2} G_{j}\left(x^{*}\right)+\sum_{k \in k_{t}} \omega_{k}^{*} \nabla^{2} H_{k}\left(x^{*}\right)\right\} z^{*}\right\rangle \\
& \geq \sum_{t=1}^{M} \tilde{\rho}_{t}\left(x, x^{*}\right)\left\|\theta\left(x, x^{*}\right)\right\|^{m} \geq-\bar{\rho}\left(x, x^{*}\right)\left\|\theta\left(x, x^{*}\right)\right\|^{m},
\end{aligned}
$$

which by virtue of (i) implies that

$$
\bar{\phi}\left(\Phi\left(x, u^{*}, v^{*}, w^{*}, \lambda^{*}\right)-\Phi\left(x, u^{*}, v^{*}, w^{*}, \lambda^{*}\right)\right) \geq 0 \text {. }
$$

The rest of the proof is identical to that of part (a). (c) and (d): The proofs are similar to those of parts (a) and (b).

\section{Theorem}

Let $\mathrm{x}^{*} \in \mathrm{F}$, let $\lambda^{*}=\phi\left(\mathrm{x}^{*}\right)$, and assume that the functions $\mathrm{f}_{\mathrm{i}}, \mathrm{g}_{\mathrm{i},}, i \in \underline{p}$ , $\mathrm{G}_{\mathrm{j}}, j \in \underline{q}$, and $\mathrm{H}_{\mathrm{k}^{\prime}}, k \in \underline{r}$, are twice differentiable at $\mathrm{x}^{*}$, and that 
for each $\mathrm{z}^{*} \in \mathrm{C}\left(\mathrm{x}^{*}\right)$, there exist $\mathrm{u}^{*} \in \mathrm{U}, v^{*} \in \mathbb{R}_{+}^{q}$, and $w^{*} \in \mathbb{R}^{r}$ such that (2.1)-(2.4) hold. Assume, furthermore, that any one of the following seven sets of hypotheses is satisfied:

a.

1. For each $i \in I_{+} \equiv\left\{i \in \underline{p}: u_{i}^{*}>0\right\}, \xi \rightarrow \Phi_{i}\left(\xi, v^{*}, w^{*}, \lambda^{*}\right)$ is $\left(F, \beta, \bar{\phi}_{i}, \pi, \bar{\rho}_{i}, \theta, m\right)$

2. Pseudosounivex at $x^{*}, \bar{\phi}_{i}$ is strictly increasing, and $\bar{\phi}_{i}(0)=0$;

3. For each , $\xi \rightarrow \Lambda_{\mathrm{t}}\left(\xi, \mathrm{v}^{*}, \mathrm{w}^{*}\right)$ is $(\mathrm{F}, \beta, \pi, \pi, \theta, \mathrm{m})$-quasisounivex $\tilde{\phi}_{t}$ at $x^{*}$, is increasing, and $\tilde{\phi}_{t}(0)=0$;

4. $\sum_{i \in I_{+}} u_{i}^{*} \bar{\rho}_{i}\left(x, x^{*}\right)+\sum_{t=1}^{M} \tilde{\rho}_{t}\left(x, x^{*}\right) \geq 0 \quad$ for all $\mathrm{x} \in \mathrm{F}$;

b.

1. For each $i \in I_{+}, \xi \rightarrow \Phi_{i}\left(\xi, v^{*}, w^{*}, \lambda^{*}\right)$ is prestrictly $\left(\mathrm{F}, \beta, \bar{\phi}_{i}, \pi\right.$, $\left.\bar{\rho}_{i}, \theta, \mathrm{m}\right)$-quasisounivex at $\mathrm{x}^{*}, \bar{\phi}_{i}$ is strictly increasing, and $\bar{\phi}_{i}$ $(0)=0$;

2. For each $t \in \underline{M}, \xi \rightarrow \Lambda_{\mathrm{t}}(\xi, \mathrm{V} *, \mathrm{~W} *)$ is strictly (F, $\beta, \bar{\phi}_{i}, \pi, \bar{\rho}_{i}$ $, \theta)$-pseudosounivex at $x^{*}$, is increasing, and $\tilde{\phi}_{t}(0)=0$;

3. $\sum_{i \in I_{+}} u_{i}^{*} \bar{\rho}_{i}\left(x, x^{*}\right)+\sum_{t=1}^{M} \tilde{\rho}_{t}\left(x, x^{*}\right) \geq 0$ for all $\mathrm{x} \in \mathrm{F}$;

c.

1. For each $i \in I_{+}, \xi \rightarrow \Phi \mathrm{i}\left(\xi, \mathrm{v}^{*}, \mathrm{w}^{*}, \lambda^{*}\right)$ is prestrictly (F, $\beta, \bar{\phi}_{i}, \pi$, $\left.\bar{\rho}_{i}, \theta, \mathrm{m}\right)$-quasisounivex at $\mathrm{x}^{*}, \bar{\phi}_{i}$ is strictly increasing, and $\bar{\phi}_{i}(0)=0$;

2. For each $t \in \underline{M}, \xi \rightarrow \Lambda_{\mathrm{t}}\left(\xi, \mathrm{v}^{*}, \mathrm{w}^{*}\right)$ is $\left(\mathrm{F}, \beta, \tilde{\phi}_{t}, \pi, \tilde{\rho}_{t}, \theta, \mathrm{m}\right)-$ quasisounivex at $\mathrm{x}^{*}$, is increasing, and $\bar{\phi}_{i}(0)=0$;

3. $\sum_{i \in I_{+}} u_{i}^{*} \bar{\rho}_{i}\left(x, x^{*}\right)+\sum_{t=1}^{M} \tilde{\rho}_{t}\left(x, x^{*}\right)>0$ for all $\mathrm{x} \in \mathrm{F}$;

d.

1. For each $i \in I_{+}, \xi \rightarrow \Phi \mathrm{i}\left(\xi, \mathrm{v}^{*}, \mathrm{w}^{*}, \lambda^{*}\right)$ is $\left(\mathrm{F}, \beta, \bar{\phi}_{i}, \pi, \tilde{\rho}_{t}, \theta, \mathrm{m}\right)$ pseudosounivex at $\mathrm{x}^{*}$, for each $i \in I_{2+}, \xi \rightarrow \Phi \mathrm{i}\left(\xi, \mathrm{v}^{*}, \mathrm{w}^{*}, \lambda^{*}\right)$ is prestrictly $(F, \beta, \pi, \theta, m)$ - quasisounivex at $x^{*}$, and for each $i \in I_{+}, \bar{\phi}_{i}$ is strictly increasing and $\bar{\phi}_{i}(0)=0$, where $\{\mathrm{I} 1+, \mathrm{I} 2+\}$ is a partition of $\mathrm{I}+$;

2. For each $t \in \underline{M}, \xi \rightarrow \Lambda \mathrm{t}\left(\xi, \mathrm{v}^{*}, \mathrm{w}^{*}\right)$ is strictly $\left(\mathrm{F}, \beta, \bar{\phi}_{i}, \pi, \tilde{\rho}_{t}\right.$ $, \theta, \mathrm{m})$-pseudosounivex at $\mathrm{x}^{*}, \tilde{\phi}_{t}$ is increasing, and $\tilde{\phi}_{t}(0)=0$;

3. $\sum_{i \in I_{+}} u_{i}^{*} \bar{\rho}_{i}\left(x, x^{*}\right)+\sum_{t=1}^{M} \tilde{\rho}_{t}\left(x, x^{*}\right) \geq 0$ for all $\mathrm{x} \in \mathrm{F}$;

e.

1. For each ${ }^{i \in I_{+}} \neq 0 \xi \rightarrow \Phi \mathrm{i}\left(\xi, \mathrm{V}^{*}, \mathrm{w}^{*}, \lambda^{*}\right)$ is $\left(\mathrm{F}, \beta, \bar{\phi}_{i}, \pi, \tilde{\rho}_{t}, \theta, \mathrm{m}\right)-$ pseudosounivex at $\mathrm{x} *$, for each $i \in I_{2+}, \xi \rightarrow \Phi \mathrm{i}\left(\xi, \mathrm{v}^{*}, \mathrm{w}^{*}, \lambda^{*}\right)$ is prestrictly $(\mathrm{F}, \beta, \pi, \theta, \mathrm{m})$-quasisounivex at $\mathrm{x}^{*}$, and for each $i \in I_{+}, \quad \tilde{\phi}_{t}$ is strictly increasing and $\tilde{\phi}_{t}(0)=0$, where $\left\{\mathrm{I}_{1}+, \mathrm{I}_{2}+\right\}$ is a partition of $\mathrm{I}_{+}$;

2. For each $t \in \underline{M}, \xi \rightarrow \Lambda_{\mathrm{t}}\left(\xi, \mathrm{v}^{*}, \mathrm{w}^{*}\right)$ is $\left(\mathrm{F}, \beta, \bar{\phi}_{i}, \pi, \tilde{\rho}_{t}, \theta, \mathrm{m}\right)$ - quasisounivex at $x^{*}, \quad \tilde{\phi}_{t}$ is increasing, and $\tilde{\phi}_{t}(0)=0$;

3. $\sum_{i \in I_{+}} u_{i}^{*} \bar{\rho}_{i}\left(x, x^{*}\right)+\sum_{t=1}^{M} \tilde{\rho}_{t}\left(x, x^{*}\right) \geq 0$ for all $\mathrm{x} \in \mathrm{F}$;

f.

1. For each $i \in I_{+}, \xi \rightarrow \Phi \mathrm{i}\left(\xi, \mathrm{v}^{*}, \mathrm{w}^{*}, \lambda^{*}\right)$ is prestrictly $\left(\mathrm{F}, \beta, \bar{\phi}_{i}, \pi\right.$, $\left.\tilde{\rho}_{t}, \theta, \mathrm{m}\right)$-quasisounivex at $\mathrm{x}^{*}$, is strictly increasing, and $\bar{\phi}_{i}$ $(0)=0$;

2. For each $t \in M_{1} \neq 0, \xi \rightarrow \Lambda_{\mathrm{t}}\left(\xi, \mathrm{V}^{*}, \mathrm{w}^{*}\right)$ is strictly $\left(\mathrm{F}, \beta, \bar{\phi}_{i}, \pi, \bar{\rho}_{i}\right.$ $, \theta, \mathrm{m})$-pseudosounivex at $\mathrm{x}^{*}$, for each $t \in \underline{M}_{2}, \xi \rightarrow \Lambda_{\mathrm{t}}\left(\xi, \mathrm{v}^{*}, \mathrm{w}^{*}\right)$ is $\left(\mathrm{F}, \beta, \bar{\phi}_{i}, \pi, \tilde{\rho}_{t}, \theta, \mathrm{m}\right)$-quasisounivex at $\mathrm{x}^{*}$, and for each $t \in \underline{M}$, is increasing and $\tilde{\phi}_{t}(0)=0$, where $\left\{M_{1}, M_{2}\right\}$ is a partition of $M$;

3. $\sum_{i \in I_{+}} u_{i}^{*} \bar{\rho}_{i}\left(x, x^{*}\right)+\sum_{t=1}^{M} \tilde{\rho}_{t}\left(x, x^{*}\right) \geq 0$ for all $\mathrm{x} \in \mathrm{F}$;

g.

1. For each ${ }^{i \in I_{+}}, \xi \rightarrow \Phi \mathrm{i}\left(\xi, \mathrm{v}^{*}, \mathrm{w}^{*}, \lambda^{*}\right)$ is $\left(\mathrm{F}, \beta, \bar{\phi}_{i}, \pi, \tilde{\rho}_{t}, \theta, \mathrm{m}\right)$ pseudosounivex at $\mathrm{x}^{*}$, for each $i \in I, \xi \rightarrow \Phi \mathrm{i}\left(\xi, \mathrm{v}^{*}, \mathrm{w}^{*}, \lambda^{*}\right)$ is prestrictly $\left(\mathrm{F}, \beta, \bar{\phi}_{i}, \pi, \tilde{\rho}_{t}, \theta, \mathrm{m}\right)-$ quasisounivex at $\mathrm{x}^{*}$, and for each $i \in I_{+}, \bar{\phi}_{i}$ is strictly increasing and $\tilde{\phi}_{t}(0)=0$, where $\left\{\mathrm{I}_{1+}\right.$, $\left.\mathrm{I}_{2+}\right\}$ is a partition of $\mathrm{I}_{+}$;

2. For each $t \in \underline{M}_{1}, \xi \rightarrow \Lambda_{\mathrm{t}}\left(\xi, \mathrm{v}^{*}, \mathrm{w}^{*}\right)$ is strictly $\left(\mathrm{F}, \beta, \bar{\phi}_{i}, \pi\right.$, $\left.\bar{\rho}_{i}, \theta, \mathrm{m}\right)$-pseudosounivex at $\mathrm{x}^{*}$, for each , $\xi \rightarrow \Lambda_{\mathrm{t}}\left(\xi, \mathrm{v}^{*}, \mathrm{w}^{*}\right)$ is $(\mathrm{F}, \beta, \pi,, \theta, \mathrm{m})$-quasisounivex $\quad$ at $\mathrm{x}^{*}$, and for each $t \in \underline{M}$ , $\bar{\phi}_{i}$ is increasing and $\bar{\phi}_{i}(0)=0$, where $\left\{\mathrm{M}_{1}, \mathrm{M}_{2}\right\}$ is a partition of $\underline{M}$;

3. $\sum_{i \in I_{+}} u_{i}^{*} \bar{\rho}_{i}\left(x, x^{*}\right)+\sum_{t=1}^{M} \tilde{\rho}_{t}\left(x, x^{*}\right) \geq 0$ for $\mathrm{M} \in \mathrm{F}$

4. $\quad I_{1+} \neq \varnothing$, or $\sum_{i \in I_{+}} u_{i}^{*} \bar{\rho}_{i}\left(x, x^{*}\right)+\sum_{t=1}^{M} \tilde{\rho}_{t}\left(x, x^{*}\right)>0$

Then $\mathrm{x}^{*}$ is an optimal solution of $(\mathrm{P})$.

\section{Proof}

(a): Suppose to the contrary that $\mathrm{x}^{*}$ is not an optimal solution of (P). Then there is a feasible solution of (P) such that $\varphi(\bar{x})<\varphi\left(x^{*}\right)=\lambda^{*}$. Hence it follows that

$$
f_{i}(\bar{x})-\lambda^{*} g_{i}(\bar{x})<0 \text { for each } i \in \underline{p} .
$$

Keeping in mind that ${ }_{v}^{*} \geq 0$, we see that for each $i \in I_{+}$, $\Phi_{i}\left(\bar{x}, v^{*}, w^{*}, \lambda^{*}\right)=f_{i}(\bar{x})-\lambda^{*} g_{i}(\bar{x})+\sum_{j \in J_{0}} v_{j}^{*} G_{j}(\bar{x})+\sum_{k \in K_{0}} w_{k}^{*} H_{k}(\bar{x})$ $\leq f_{i}(\bar{x})-\lambda^{*} g_{i}(\bar{x})$ (by the feasibility of $\bar{x}$ ) $<0$ $f_{i}(\bar{x})-\lambda^{*} g_{i}(\bar{x})+\sum_{j \in J_{0}} v_{j}^{*} G_{j}(\bar{x})+\sum_{k \in K_{0}} w_{k}^{*} H_{k}(\bar{x})$

(by (2.3), (2.4), and the feasibility of $x^{*}$ )

$=\Phi_{i}\left(x^{*}, v^{*}, w^{*}, \lambda^{*}\right)$

and so using the properties of the function $\bar{\phi}_{i}$, we get $\phi\left(\Phi_{i}\left(\bar{x}, v^{*}, w^{*}, \lambda^{*}\right)-\Phi_{i}\left(x^{*}, v^{*}, w^{*}, \lambda^{*}\right)\right)<0$

which in view of (i) implies that 
$F\left(\bar{x}, x^{*} ; \beta\left(\bar{x}, x^{*}\right)\left[\nabla f_{i}\left(x^{*}\right)-\lambda * \nabla g_{i}\left(x^{*}\right)+\sum_{i \in J_{0}} v_{j}^{*} \nabla G_{j}\left(x^{*}\right)+\sum_{k \in K_{0}} \omega_{k}^{*} \nabla H_{k}\left(x^{*}\right)\right]\right)$

$+\left\langle\pi\left(\bar{x}, x^{*}\right),\left[\nabla^{2} f_{i}\left(x^{*}\right)-\lambda^{*} \nabla^{2} g_{i}\left(x^{*}\right)+\sum_{i \in J_{t}} v_{j}^{*} \nabla^{2} G_{j}\left(x^{*}\right)+\sum_{k \in k_{t}} \omega_{k}^{*} \nabla^{2} H_{k}\left(x^{*}\right)\right] z^{*}\right\rangle$

$-\frac{1}{2}\left\langle z^{*},\left[\nabla^{2} f_{i}\left(x^{*}\right)-\lambda^{*} \nabla^{2} g_{i}\left(x^{*}\right)+\sum_{i \in J_{t}} v_{j}^{*} \nabla^{2} G_{j}\left(x^{*}\right)+\sum_{k \in k_{t}} \omega_{k}^{*} \nabla^{2} H_{k}\left(x^{*}\right)\right] z^{*}\right\rangle$

$-\bar{\rho}\left(x, x^{*}\right)\left\|\theta\left(x, x^{*}\right)\right\|^{m}$

Since $u^{*}>0, u_{i}^{*}=0$ for each $i \in \underline{p} \backslash I_{+}, \sum_{i=1}^{p} u_{i}^{*}=1$ and

$F\left(x, x^{*}, \cdot\right)$ is sublinear, the above inequalities yield

$f\left(\bar{x}, x^{*} ; \beta\left(\bar{x}, x^{*}\right)\left\{\sum_{i=1}^{p} \mu_{i}^{*}\left[\nabla f_{i}\left(x^{*}\right)-\lambda^{*} \nabla_{g i}\left(x^{*}\right)\right]+\sum_{j \in j_{o}} v_{j}^{*} \nabla G_{j}\left(x^{*}\right)+\sum_{k \in k_{o}} w_{k}^{*} \nabla H_{k}\left(x^{*}\right)\right\}\right.$

$+\left\langle\pi\left(\bar{x}, x^{*}\right),\left\{\sum_{i=1}^{p} \mu_{i}^{*}\left[\nabla^{2} f_{i}\left(x^{*}\right)-\lambda^{*} \nabla^{2} g i\left(x^{*}\right)\right]+\sum_{j \in j_{0}} v_{j}^{*} \nabla^{2} G_{j}\left(x^{*}\right)+\sum_{k \in k_{0}} w_{k}^{*} \nabla^{2} H_{k}\left(x^{*}\right)\right\} z^{*}\right\rangle$

$-\frac{1}{2}\left\langle z^{*},\left\{\sum_{i=1}^{p} \mu_{i}^{*}\left[\nabla^{2} f_{i}\left(x^{*}\right)-\lambda^{*} \nabla^{2} g_{i}\left(x^{*}\right)\right]+\sum_{j \in j_{0}} v_{j}^{*} \nabla^{2} G_{j}\left(x^{*}\right)+\sum_{k \in k_{0}} w_{k}^{*} \nabla^{2} H_{k}\left(x^{*}\right)\right\} z^{*}\right\rangle$

$<-\sum_{i \in I_{+}} \mu_{i}^{*} \bar{p}_{i}\left(x, x^{*}\right)\left\|\theta\left(\bar{x}, x^{*}\right)\right\|^{m}$

Proceeding as in the proof of Theorem 2.1, we see that our assumptions in (ii) lead to

$$
\begin{gathered}
\beta\left(\bar{x}, x^{*}\right) \sum_{t=1}^{m}\left[\sum_{j \in j_{i}} v_{j}^{*} \nabla G_{j}\left(x^{*}\right)+\sum_{k \in k_{t}} w_{k}^{*} \nabla H_{k}\left(x^{*}\right)\right]+\left\langle\pi\left(\bar{x}, x^{*}\right),\right. \\
\left.\sum_{t=1}^{m}\left[\sum_{j \in j_{i}} v_{j}^{*} \nabla^{2} G_{j}\left(x^{*}\right)+\sum_{k \in k_{t}} w_{k}^{*} \nabla^{2} H_{k}\left(x^{*}\right)\right] z^{*}\right\rangle \\
-\frac{1}{2}\left\langle z^{*}, \sum_{t=1}^{m}\left[\sum_{j \in j_{t}} v_{j}^{*} \nabla^{2} G_{j}\left(x^{*}\right)+\sum_{k \in k_{i}} w_{k}^{*} \nabla^{2} H_{k}\left(x^{*}\right)\right] z^{*}\right\rangle \leq-\sum_{t=1}^{m} \tilde{p}_{t}\left(x, x^{*}\right)\left\|\theta\left(\bar{x}, x^{*}\right)\right\|^{2}
\end{gathered}
$$

which when combined with (2.5) and (2.6) results in

$f\left(\bar{x}, x^{*} ; \beta\left(\bar{x}, x^{*}\right)\left\{\sum_{i=1}^{p} \mu_{i}^{*}\left[\nabla f_{i}\left(x^{*}\right)-\lambda^{*} \nabla g_{i}\left(x^{*}\right)\right]+\sum_{j \in j_{0}} v_{j}^{*} G_{j}\left(x^{*}\right)+\sum_{k \in \epsilon_{0}} w_{k}^{*} \nabla H_{k}\left(x^{*}\right)\right\}\right)$

$+\left\langle\pi\left(\bar{x}, x^{*}\right),\left\{\sum_{t=1}^{p} \mu_{i}^{*}\left[\nabla^{2} f_{i}\left(x^{*}\right)-\lambda^{*} \nabla^{2} g_{i}\left(x^{*}\right)\right]+\sum_{j \in j_{0}} v_{j}^{*} \nabla^{2} G_{j}\left(x^{*}\right)+\sum_{k \in k_{0}} w_{k}^{*} \nabla^{2} H_{k}\left(x^{*}\right)\right\} z^{*}\right\rangle$

$-\frac{1}{2}\left\langle z^{*},\left\{\sum_{t=1}^{p} \mu_{i}^{*}\left[\nabla^{2} f_{i}\left(x^{*}\right)-\lambda^{*} \nabla^{2} G_{j}\left(x^{*}\right)+\sum_{k \in k_{0}} w_{k}^{*} \nabla^{2} H_{k}\left(x^{*}\right)\right]\right\} z^{*}\right\rangle$

$\geq \sum_{t=1}^{m} \tilde{p}_{t}\left(x, x^{*}\right)\left\|\theta\left(\bar{x}, x^{*}\right)\right\|^{2}$

In view of (iii), this inequality contradicts (2.11). Hence, $\mathrm{X} *$ is an optimal solution of $(\mathrm{P})$.

(b)-(g): The proofs are similar to that of part (a).

In the next theorem, we make use of a slightly different partitioning method which appears to have been used for the first time by Yang [3] for the purpose of formulating a general duality model for a multi objective fractional programming problem, to present another collection of sufficient optimality results for (P) which are somewhat different from those stated in Theorems 2.1 and 2.2. These results are formulated by utilizing a partition of in addition to those of and, and by placing appropriate generalized $(F, \beta, \phi, \Pi, \rho, \theta, m)$-sounivexity requirements on certain combinations of the problem functions.
Let $\left\{I_{o}, I_{1}, \ldots, I_{l}\right\} \quad$ be a partition of $\mathrm{p}$ such that $l=\{0,1,2, \ldots, l\} \quad \subset M=\{\mathbf{O}, \mathbf{1}, \ldots, M\}$, and let the real-valued function $\xi \rightarrow \prod_{t}(\xi, u, v, w, \lambda)$ be defined, for fixed $u, v, w$, and $\lambda$, by

$\Pi_{t}(\xi, u, v, w, \lambda)=\sum_{i \in I_{t}} u_{i}\left[f_{i}(\xi)-\lambda g_{i}(\xi)\right]+\sum_{j \in J_{t}} v_{j} G_{j}(\xi)+\sum_{k \in K_{t}} w_{k} H_{k}(\xi), t \in \underline{M}$.

Theorem

Let $x^{*} \in \mathrm{F}$, let $\lambda^{*}=\varphi\left(x^{*}\right)$, and assume that the functions $f_{i}, g_{i}, i \in \underline{p}, G_{j}, j \in \underline{q}$,

and $H_{k}, k \in \underline{r}$, are twice differentiable at $\mathrm{x}^{*}$, and that for each $z^{*} \in C\left(x^{*}\right)$, there exist $u^{*} \in U, v^{*} \in \mathbb{R}^{q}$, and $w^{*} \in \mathbb{R}^{r}$ such that (2.1)-(2.4) hold. Assume, furthermore, that any one of the following seven sets of hypotheses is satisfied:

a.

1. For each $t \in \Gamma^{2} \xi \rightarrow \amalg^{\mathrm{t}}\left(\mathcal{2}^{2} N_{*}{ }^{2} \wedge_{*}{ }^{2} M_{*}{ }^{2} Y_{*}\right)$ is strictly $\left(F, \beta, \phi_{t}, \pi, \rho_{t}, \theta, m\right)$-pseudosounivex at $x^{*}, \phi_{t}$ is increasing, and $\phi_{t}(0)=0$;

2. For each $t \in M \backslash L, \xi \rightarrow \Lambda_{t}\left(\xi, v^{*}, w^{*}, \lambda^{*}\right)$ is $\left(F, \beta, \phi_{t}, \pi, \rho_{t}, \theta, m\right)$-quasisounivex at $x^{*}, \phi_{t}$ is increasing, and $; \phi_{t}(0)=0$

3. $\sum_{t \in M} \rho_{t}\left(x, x^{*}\right) \geq 0$ for all $x \in \mathbb{F}$;

b.

1. For each $t \in L, \quad \xi \rightarrow \prod_{t}\left(\xi, u^{*}, v^{*}, w^{*}, \lambda^{*}\right)$ is prestrictly $\left(F, \beta, \phi_{t}, \pi, \rho_{t}, \theta, m\right)^{-}$quasisounivex at is increasing, and ; $\phi_{t}(0)=0$

2. For each $t \in M \backslash L, \xi \rightarrow \Lambda_{t}\left(\xi, v^{*}, w^{*}, \lambda^{*}\right)$ is strictly $\left(F, \beta, \phi_{t}, \pi, \rho_{t}, \theta, m\right)$-pseudosounivex at $x^{*}, \phi_{t}$ is increasing, and $\phi_{t}(0)=0$;

3. $\sum_{t \in M} \rho_{t}\left(x, x^{*}\right) \geq 0$ for all $x \in \mathbb{F}$;

c.

1. for each $t \in L, \xi \rightarrow \prod_{t}\left(\xi, u^{*}, v^{*}, w^{*}, \lambda^{*}\right)$ is prestrictly $\left(F, \beta, \phi_{t}, \pi, \rho_{t}, \theta, m\right)$-quasisounivex at $x^{*}, \phi_{t}$ is increasing, and $\phi_{t}(0)=0$;

2. for each $t \in M \backslash L, \xi \rightarrow \Lambda_{t}\left(\xi, v^{*}, w^{*}, \lambda^{*}\right)$ is -quasisounivex at

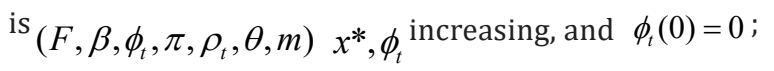

3. $\sum_{t \in M} \rho_{t}\left(x, x^{*}\right) \geq 0$ for all $x \in \mathbb{F}$;

d.

1. For each $t \in L_{1}, \quad \xi \rightarrow \prod_{t}\left(\xi, u^{*}, v^{*}, w^{*}, \lambda^{*}\right)$ is strictly $\left(F, \beta, \phi_{t}, \pi, \rho_{t}, \theta, m\right)$-pseudosounivex at $\mathrm{x}^{*}$, for each $t \in L_{2}$ , $\xi \rightarrow \prod_{t}\left(\xi, u^{*}, v^{*}, w^{*}, \lambda^{*}\right)$ is prestrictly $\left(F, \beta, \phi_{t}, \pi, \rho_{t}, \theta, m\right)$ -quasisounivex at $\mathrm{x}^{*}$, and for each $t \in L$, is increasing and , $\phi_{t}(0)=0$ where $\{\mathrm{L} 1, \mathrm{~L} 2\}$ is a partition of $\mathrm{L}$; 
2. For each $t \in M \backslash L, \xi \rightarrow \Lambda_{t}\left(\xi, v^{*}, w^{*}, \lambda^{*}\right)$ is strictly $\left(F, \beta, \phi_{t}, \pi, \rho_{t}, \theta, m\right)$-pseudosounivex at $\mathrm{x}^{*}, \quad$ is increasing, and $\phi_{t}(0)=0$;

3. $\sum_{t \in M} \rho_{t}\left(x, x^{*}\right) \geq 0$ for all $x \in \mathbb{F}$;

e.

1. For each $t \in L_{1} \neq 0, \xi \rightarrow \prod_{t}\left(\xi, u^{*}, v^{*}, w^{*}, \lambda^{*}\right)$ is strictly $\left(F, \beta, \phi_{t}, \pi, \rho_{t}, \theta, m\right)$ - pseudosounivex at $\mathrm{x}^{*}$, for each $t \in L_{2}$ $\xi \rightarrow \prod_{t}\left(\xi, u^{*}, v^{*}, w^{*}, \lambda^{*}\right)$, is prestrictly $\left(F, \beta, \phi_{t}, \pi, \rho_{t}, \theta, m\right)$ -quasisounivex at $\mathrm{x}^{*}$, and for each $t \in L$, is increasing and $\phi_{t}(0)=0$, where $\{L 1, L 2\}$ is a partition of L;

2. For each $t \in M \backslash L, \xi \rightarrow \Lambda_{t}\left(\xi, v^{*}, w^{*}, \lambda^{*}\right) \quad$ is $\left(F, \beta, \phi_{t}, \pi, \rho_{t}, \theta, m\right)$-quasisounivex at $\mathrm{x}^{*}, \phi_{t}$ is increasing, and; $\phi_{t}(0)=0$

3. $\sum_{t \in M} \rho_{t}\left(x, x^{*}\right) \geq 0$ for all $x \in \mathbb{F}$;

f.

1. Foreach $t \in L \xi \rightarrow \prod_{t}\left(\xi, u^{*}, v^{*}, w^{*}, \lambda^{*}\right)$, is prestrictly $\left(F, \beta, \phi_{t}, \pi, \rho_{t}, \theta, m\right)$-quasisounivex at $\mathrm{x}^{*}$, is increasing, and ; $\phi_{t}(0)=0$

2. For each $t \in(M \backslash L)_{1} \neq \phi, t \in M \backslash L, \xi \rightarrow \Lambda_{t}\left(\xi, v^{*}, w^{*}\right)$ is strictly $\left(F, \beta, \phi_{t}, \pi, \rho_{t}, \theta, m\right)$ pseudosounivex at $\mathrm{x}^{*}$, for each $t \in(M \backslash L)_{2}, \quad \xi \rightarrow \Lambda_{t}\left(\xi, v^{*}, w^{*}\right) \quad$ is $\left(F, \beta, \phi_{t}, \pi, \rho_{t}, \theta, m\right)$ quasisounivex at $\mathrm{x}^{*}$, and for each $\mathrm{t} \in \mathrm{L}$, is increasing $\phi_{t}(0)=0$ and, where $\left\{(M \backslash L)_{1},(M \backslash L)_{2}\right\}$ is a partition of $M \backslash L$;

3. $\sum_{t \in M} \rho_{t}\left(x, x^{*}\right) \geq 0$ for all $\mathrm{x} \in \mathrm{F}$;

g.

1. For each $t \in L_{1}, \quad \xi \rightarrow \prod_{t}\left(\xi, u^{*}, v^{*}, w^{*}, \lambda^{*}\right) \quad$ is $\left(F, \beta, \phi_{t}, \pi, \rho_{t}, \theta, m\right)$-pseudosounivex at $\mathrm{x}^{*}$, for each $t \in L_{2}, \quad \xi \rightarrow \prod_{t}\left(\xi, u^{*}, v^{*}, w^{*}, \lambda^{*}\right) \quad$ is prestrictly $\left(F, \beta, \phi_{t}, \pi, \rho_{t}, \theta, m\right)$-quasisounivex at $\mathrm{x}^{*}$, and for each $\mathrm{t} \in \mathrm{L}$, is increasing and, where $\left\{\mathrm{L}_{1}, \mathrm{~L}_{2}\right\}$ is a partition of $\mathrm{L}$;

2. For each $t \in M \backslash L, \xi \rightarrow \Lambda_{t}\left(\xi, v^{*}, w^{*}\right)$ is strictly $\left(F, \beta, \phi_{t}, \pi, \rho_{t}, \theta, m\right)$-pseudosounivex at $\mathrm{x}^{*}$, for each $t \in(M \backslash L)_{2} \rightarrow \Lambda_{t}\left(\xi, v^{*}, w^{*}\right)$ is $\left(F, \beta, \phi_{t}, \pi, \rho_{t}, \theta, m\right)$-quasisounivex at $\mathrm{X}^{*}$, and for each , is increasing and $(0)=0$, where $\left\{(\mathrm{M} \backslash \mathrm{L})_{1}\right.$, $\left.(\mathrm{M} \backslash \mathrm{L})_{2}\right\}$ is a partition of $\mathrm{L}$;

3. $\sum_{t \in M} \rho_{t}\left(x, x^{*}\right) \geq 0$ for all $\mathrm{x} \in \mathrm{F}$;

4. $L_{1} \neq 0,(M \backslash L)_{1} \neq 0$, or $\sum_{t \in M} \rho_{t}\left(x, x^{*}\right)>0$.

Then $\mathrm{x}^{*}$ is an optimal solution of $(\mathrm{P})$.

\section{Proof (a)}

Suppose to the contrary that $\mathrm{x} *$ is not an optimal solution of (P). This implies that

$$
f_{i}(\bar{x})-\lambda^{*} g_{i}(\bar{x})<0, \quad i \in \underline{p},
$$

for some $\bar{x} \in F$. Since $u^{*} \geq 0$ and $u^{*} \neq 0$, we see that for each $t \in L$,

$\sum_{i \in I_{t}} u_{i}\left[f_{i}(\bar{x})-\lambda g_{i}(\bar{x})\right] \leq 0$

Now using this inequality, we see that

$\Pi_{t}\left(\bar{x}, u^{*} ; v^{*}, \omega^{*}, \lambda^{*}\right)=\sum_{i \in I_{t}} u_{i}^{*}\left[f_{i}(\bar{x})-\lambda * g_{i}(\bar{x})\right]+\sum_{j \in J_{t}} v_{j}^{*} G_{j}(\bar{x})+\sum_{k \in K_{t}} w_{k}^{*} H_{k}(\bar{x})$

$\leq \sum_{i \in i_{T}} u_{i}^{*}\left[f_{i}(\bar{x})-\lambda * g_{i}(\bar{x})\right]$ (by the feasibility of $\bar{x}$ )

$\leq 0$ (by (2.12))

$=\sum_{i \in I_{t}} u_{i}^{*}\left[f_{i}(\bar{x})-\lambda * g_{i}(\bar{x})\right]+\sum_{j \in J_{t}} v_{j}^{*} G_{j}(\bar{x})+\sum_{k \in K_{t}} w_{k}^{*} H_{k}\left(x^{*}\right)$

(by (2.3), (2.4), and the feasibility of $x^{*}$ )

$$
=\Pi_{t}\left(x^{*}, u^{*}, v^{*}, w^{*}, \lambda^{*}\right)
$$

and hence $\Phi_{t}\left(\Pi_{t}\left(x^{*}, u^{*}, v^{*}, w^{*}, \lambda^{*}\right)-\Pi_{t}\left(x^{*}, u^{*}, v^{*}, w^{*}, \lambda^{*}\right)\right) \leq 0$,

which in view of (i) implies that

$F\left(\bar{x}, x^{*} ; \beta\left(\bar{x}, x^{*}\right)\left\{\sum_{i \in I_{t}} u_{i}^{*}\left[\nabla f_{i}\left(x^{*}\right)-\lambda * \nabla g_{i}\left(x^{*}\right)\right]+\sum_{j \in J_{t}} v_{j}^{*} \nabla G_{j}\left(x^{*}\right)+\sum_{j \in J_{t}} w_{j}^{*} \nabla H_{k}\left(x^{*}\right)\right\}\right)$

$+\left\langle\pi\left(\bar{x}, x^{*}\right),\left\{\sum_{i \in I_{t}} u_{i}^{*}\left[\nabla^{2} f_{i}\left(x^{*}\right)\right]+\sum_{j \in J_{t}} v_{j}^{*} \nabla^{2} G_{j}\left(x^{*}\right)+\sum_{k \in K_{t}} w_{k}^{*} \nabla^{2} H_{k}\left(x^{*}\right) z^{*}\right\}\right\rangle$

$-\frac{1}{2}\left\langle z^{*},\left\{\sum_{i \in l_{t}} u_{i}^{*}\left[\nabla^{2} g_{i}\left(x^{*}\right)\right]+\sum_{j \in J_{t}} v_{j}^{*} \nabla^{2} G_{j}\left(x^{*}\right)+\sum_{k \in K_{i}} w_{k}^{*} \nabla^{2} H_{k}\left(x^{*}\right)\right\} z^{*}\right\rangle<-\rho_{t}\left(\bar{x}, x^{*}\right)\left\|\theta\left(\bar{x}, x^{*}\right)\right\|^{m}$.

Summing over and using the sublinearity of $F\left(x, x^{*} ; \therefore\right.$, we obtain

$F\left(\bar{x}, x^{*} ; \beta\left(\bar{x}, x^{*}\right)\left\{\sum_{i=1} u_{i}^{*}\left[\nabla f_{i}\left(x^{*}\right)-\lambda^{*} \nabla g_{i}\left(x^{*}\right)\right]+\sum_{i \in L}\left[\sum_{j \in J_{t}} v_{j}^{*} \nabla G_{j}\left(x^{*}\right)+\sum_{k \in K_{t}} w_{k}^{*} \nabla H_{k}\left(x^{*}\right)\right]\right\}\right)$

$\left.+\left\langle\pi\left(\bar{x}, x^{*}\right), \sum_{t \in L}\left[\sum_{i \in I_{i}} u_{i}^{*}\left[\nabla^{2} f_{i}\left(x^{*}\right)-\lambda^{*} \nabla^{2} g_{i}\left(x^{*}\right)\right]+\right]+\sum_{j \in J_{t}} v_{j}^{*} \nabla^{2} G_{j}\left(x^{*}\right)+\sum_{k \in K_{t}} w_{k}^{*} \nabla^{2} H_{k}\left(x^{*}\right)\right\} z^{*}\right\rangle$

$\left.-\frac{1}{2}\left\langle z^{*}, \sum_{t \in L}\left\{\sum_{i \in l_{t}} u_{i}^{*}\left[\nabla^{2} f_{i}\left(x^{*}\right)-\lambda^{*} \nabla^{2} g_{i}\left(x^{*}\right)\right]+\sum_{j \in J_{t}} v_{j}^{*} \nabla^{2} G_{j}\left(x^{*}\right)+\sum_{k \in K_{t}} w_{k}^{*} \nabla^{2} H_{k}\left(x^{*}\right)\right\}\right\} z^{*}\right\rangle$

$<-\sum_{t \in L} \rho_{t}\left(\bar{x}, x^{*}\right)\left\|\theta\left(\bar{x}, x^{*}\right)\right\|^{m} \cdot(2.13)$

As shown in the proof of Theorem 2.1, for each $t \in M / L$, $\Lambda_{t}\left(\bar{x}, v^{*}, w^{*}\right) \leq \Lambda_{t}\left(x^{*}, v^{*}, w^{*}\right)$,

and so

$\phi_{t}\left(\Lambda_{t}\left(\bar{x}, v^{*}, w^{*}\right)-\Lambda_{t}\left(x^{*}, v^{*}, w^{*}\right) \leq 0\right.$

which in view of (ii) implies that

$\phi_{t}\left(\Lambda_{t}\left(\bar{x}, v^{*}, w^{*}\right)-\Lambda_{t}\left(x^{*}, v^{*}, w^{*}\right) \leq 0\right.$

$+\left\langle\pi\left(\bar{x}, x^{*}\right),\left[\sum_{j \in J_{t}} v_{j}^{*} \nabla G_{j}\left(x^{*}\right)+\sum_{k \in K_{t}} w_{k}^{*} \nabla H_{k}\left(x^{*}\right)\right] z^{*}\right\rangle$

$-\frac{1}{2}\left\langle z^{*},\left(\bar{x}, x^{*}\right),\left[\sum_{j \in J_{t}} v_{j}^{*} \nabla G_{j}\left(x^{*}\right)+\sum_{k \in K_{t}} w_{k}^{*} \nabla H_{k}\left(x^{*}\right)\right] z^{*}\right\rangle$

Summing over $t \in M / L$, we get

$F\left(\bar{x}, x^{*} ; \beta\left(\bar{x}, x^{*}\right) \sum_{t \in M \backslash L}\left[\sum_{j \in J_{t}} v_{j}^{*} \nabla G_{j}\left(x^{*}\right)+\sum_{k \in K_{t}} w_{k}^{*} \nabla H_{k}\left(x^{*}\right)\right]\right)$

$+\left\langle\pi\left(\bar{x}, x^{*}\right), \sum_{t \in M \backslash L}\left[\sum_{j \in J_{t}} v_{j}^{*} \nabla G_{j}\left(x^{*}\right)+\sum_{k \in K_{t}} w_{k}^{*} \nabla H_{k}\left(x^{*}\right)\right] z^{*}\right\rangle$ 


$$
\begin{aligned}
& -\frac{1}{2}\left\langle z^{*}, \sum_{t \in M \backslash L}\left[\sum_{j \in J_{t}} v_{j}^{*} \nabla G_{j}\left(x^{*}\right)+\sum_{k \in K_{t}} w_{k}^{*} \nabla H_{k}\left(x^{*}\right)\right] z^{*}\right\rangle \\
& <-\sum_{t \in M \backslash L} \rho_{t}\left(\bar{x}, x^{*}\right)\left\|\theta\left(\bar{x}, x^{*}\right)\right\|^{m} .
\end{aligned}
$$

Combining (2.13) and (2.14) and using (iii), we obtain $F\left(\bar{x}, x^{*} ; \beta\left(\bar{x}, x^{*}\right)\left\{\sum_{i=1}^{p} u_{i}^{*}\left[\nabla f_{i}\left(x^{*}\right)-\lambda^{*} \nabla g_{i}\left(x^{*}\right)\right]+\sum_{j=1}^{q} u_{j}^{*} \nabla G_{j}\left(x^{*}\right)+\sum_{k=1}^{r} w_{k}^{*} \nabla H_{k}\left(x^{*}\right)\right\}\right)$ $+\left\langle\pi\left(\bar{x}, x^{*}\right),\left\{\sum_{i=1}^{p} u_{i}^{*}\left[\nabla f_{i}\left(x^{*}\right)-\lambda^{*} \nabla g_{i}\left(x^{*}\right)\right]+\sum_{j=1}^{q} u_{j}^{*} \nabla G_{j}\left(x^{*}\right)+\sum_{k=1}^{r} w_{k}^{*} \nabla H_{k}\left(x^{*}\right)\right\} z^{*}\right\rangle$ $-\frac{1}{2}\left\langle z^{*},\left\{\sum_{i=1}^{p} u_{i}^{*}\left[\nabla f_{i}\left(x^{*}\right)-\lambda * \nabla g_{i}\left(x^{*}\right)\right]+\sum_{j=1}^{q} u_{j}^{*} \nabla G_{j}\left(x^{*}\right)+\sum_{k=1}^{r} w_{k}^{*} \nabla H_{k}\left(x^{*}\right)\right\} z^{*}\right\rangle$ $<-\sum_{t \in M} \rho_{t}\left(\bar{x}, x^{*}\right)\left\|\theta\left(v, x^{*}\right)\right\|^{m} \leq 0$,

Now if we multiply (2.1) by $\beta\left(\bar{x}, x^{*}\right)$, apply $F\left(x, x^{*} ;\right)$ to both sides of the resulting equation, using the sublinearity of $F\left(x, x^{*} ; \cdot\right)$, and adding this equation to (2.2), we obtain the following inequality:

$$
\begin{aligned}
& F\left(\bar{x}, x^{*} ; \beta\left(\bar{x}, x^{*}\right)\left\{\sum_{i=1}^{p} u_{i}^{*}\left[\nabla f_{i}\left(x^{*}\right)-\lambda^{*} \nabla g_{i}\left(x^{*}\right)\right]+\sum_{j=1}^{q} v_{i}^{*} \nabla G_{j}\left(x^{*}\right)+\sum_{k=1}^{r} w_{k}^{*} \nabla H_{k}\left(x^{*}\right)\right\}\right) \\
& +\left\langle\pi\left(x, x^{*}\right), \sum_{i=1}^{p} u_{i}^{*}\left[\nabla^{2} f_{i}\left(x^{*}\right)-\lambda^{*} \nabla^{2} g_{i}\left(x^{*}\right)\right]+\sum_{j=1}^{q} v_{j}^{*} \nabla^{2} G_{j}\left(x^{*}\right)+\sum_{k=1}^{r} w_{k}^{*} \nabla^{2} H_{k}\left(x^{*}\right) z^{*}\right\rangle \\
& -\frac{1}{2}\left[\left\langlez^{*},\left\{\sum_{i=1}^{p} u_{i}^{*}\left[\nabla^{2} f_{i}\left(x^{*}\right)-\lambda^{*} \nabla^{2} g_{i}\left(x^{*}\right)+\sum_{j=1}^{q} v_{j}^{*} \nabla^{2} G_{j}\left(x^{*}\right)+\sum_{k=1}^{r} w_{k}^{*} \nabla^{2} H_{k}\left(x^{*}\right) z^{*}\right\rangle\right] \geq 0\right.\right.
\end{aligned}
$$

\section{Concluding Remarks}

Based on Dinkelbach's parametric model [1], we have established numerous sets of higher order parametric sufficient optimality criteria for a discrete minmax fractional programming problem using a variety of generalized $(F, \beta, \phi, \pi, \rho, \theta, m)$ -sounivexity constraints. These optimality models can be applied for constructing various duality models as well as for developing new algorithms for the numerical solution of minmax fractional programming problems. Furthermore, the obtained results generalize most of the results available in the current literature, and are application-oriented in the sense of interdisciplinary research. More importantly, duality models do have a significant role in the semiinfinite aspects of the mathematical programming, for example, the following semiinfinite minmax fractional programming problem:

$$
\text { Minimize } \max _{1 \leq i \leq p} \frac{f_{i}(x)}{g_{i}(x)}
$$

subject to $G_{j}(x, t) \leq 0$ for all $t \in T_{j}, j \in \underline{q} ; H_{k}(x, s)=0$ for all $s \in S_{k}, k \in \underline{r} ; x \in X$, where $\mathrm{X}$, fi, and gi, , are as defined in the description of (P), for each $j \in \underline{q} \quad \xi \rightarrow G_{j}(\xi, t)$ and , Tj and Sk are compact subsets of complete metric spaces, for each , is a real-valued function defined on X for all $t \in T_{j}, \xi \rightarrow H_{k}(\xi, s)$ for each $j \in \underline{q}$, is a real-valued function defined on $\mathrm{X}$ for all , for each $k \in \underline{r} \quad t \rightarrow G_{j}(x, t)$ and $s \rightarrow H_{k}(x, s)$, and are continuous realvalued functions defined, respectively, on $\mathrm{T}_{\mathrm{j}}$ and $\mathrm{S}_{\mathrm{k}}$ for all $\mathrm{x} \in \mathrm{X}$.

\section{References}

1. Hanson MA (1993) Second order invexity and duality in mathematical programming. Opsearch 30: 313-320.

2. Mond B, Weir T (1981) Generalized concavity and duality. In: Schaible S \& Ziemba WT (Eds.), Generalized Concavity in Optimization and Economics, Academic Press, New York, USA, pp. 263-279.

3. Yang X (1994) Generalized convex duality for multi objective fractional programs. Opsearch 31: 155-163.

4. Dinkelbach W (1967) On nonlinear fractional programming. Management Sci 13: 492- 498.

5. Verma RU, Zalmai GJ (2016) Second-order parametric optimality conditions in discrete minmax fractional programming. Communications on Applied Nonlinear Analysis 23(3): 1-32.

6. Verma RU, Zalmai GJ (2016) Second order parametric optimality conditions in semi-infinite discrete minmax fractional programming based on second-order sonvexities. Transactions on Mathematical Programming and Applications 4(1): 62- 84.

7. Zalmai GJ (1989) Optimality conditions and duality for constrained measurable subset selection problems with minmax objective functions, Optimization 20: 377-395.

8. Zalmai GJ (2012) Generalized second-order $(F, \beta, \varphi, \rho, \theta)$-univex functions and parametric duality models in semiinfinite discrete minmax fractional programming, Advances in Nonlinear Variational Inequalities 15: 63-91.

9. Zalmai GJ, Zhang Q (2007) Generalized (F, $\beta, \varphi, \rho, \theta)$-univex functions and global parametric sufficient optimality conditions in semiinfinite discrete minmax fractional programming, Pan American Mathematical Journal 17: 1-26.

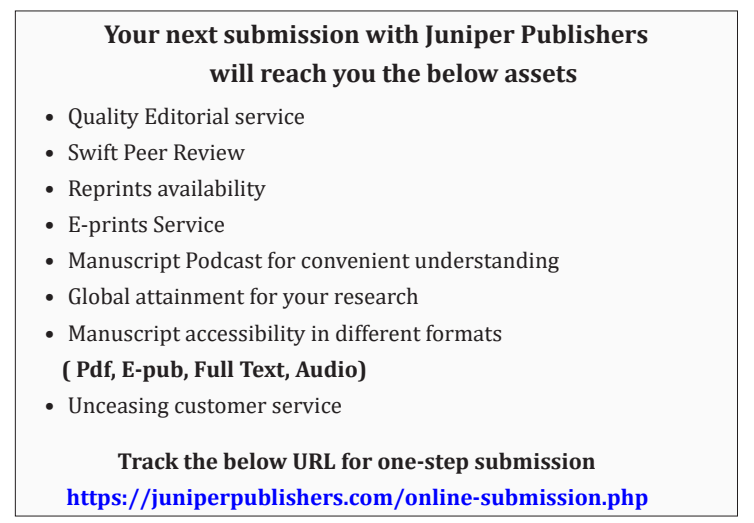

